\title{
Radiation Damage to Nervous System: Designing Optimal Models for Realistic Neuron Morphology in Hippocampus
}

\author{
Munkhbaatar Batmunkh ${ }^{1,2, \star}$, Alexander Bugay ${ }^{1}$, Lkhagvaa Bayarchimeg ${ }^{1}$, and Oidov Lkhagva ${ }^{2}$ \\ ${ }^{1}$ Laboratory of Radiation Biology, Joint Institute for Nuclear Research, 141980, Dubna \\ ${ }^{2}$ Division of Natural Sciences, National University of Mongolia, 210646, Ulaanbaatar
}

\begin{abstract}
The present study is focused on the development of optimal models of neuron morphology for Monte Carlo microdosimetry simulations of initial radiation-induced events of heavy charged particles in the specific types of cells of the hippocampus, which is the most radiation-sensitive structure of the central nervous system. The neuron geometry and particles track structures were simulated by the Geant4/Geant4-DNA Monte Carlo toolkits. The calculations were made for beams of protons and heavy ions with different energies and doses corresponding to real fluxes of galactic cosmic rays. A simple compartmental model and a complex model with realistic morphology extracted from experimental data were constructed and compared. We estimated the distribution of the energy deposition events and the production of reactive chemical species within the developed models of CA3/CA1 pyramidal neurons and DG granule cells of the rat hippocampus under exposure to different particles with the same dose. Similar distributions of the energy deposition events and concentration of some oxidative radical species were obtained in both the simplified and realistic neuron models.
\end{abstract}

\section{Introduction}

Recent experimental studies at particle accelerators have shown the occurrence of neurochemical alterations, oxidative and radical damage, modifications to dendritic morphology, electrophysiological alterations in different parts of the brain of the laboratory animals after exposure to energetic protons and heavy ion beams at doses matching the real fluxes in the energy spectrum of galactic cosmic rays and solar particle events [1-3]. The analysis of these measurements suggests that one of the most radiation sensitive-brain structure is the hippocampus. The hippocampus plays an important role in learning, memory consolidation as well as the integration of the information received simultaneously from diverse sources. The precise mechanisms of the radiation-induced brain injury remains mostly unclear. The development of models of the radiation-induced structural and functional variations in different brain structures (especially the hippocampus) is important for the understanding of the longterm changes to the neuronal electrophysiological states as a result of prolonged deep space missions [3]. The present work focuses at designing an optimal model of the hippocampal neurons irradiation with the aim at understanding the electrophysiological behaviour of the hippocampal neural network.

\footnotetext{
^e-mail: batmunkh@jinr.ru
} 


\section{Simplified neuron models in the Monte Carlo track-structure simulations}

Neuronal cells exist in different shapes and sizes, and can be classified by their morphology and function. In the different regions of hippocampus, there are two main types of neuronal cells: 1.2 million granule cells in dentate gyrus region (DG) and 615000 pyramidal cells in cornu ammonis region (CA3/CA1). A soma of a granule cell rests in the cell layer of the DG region, while its dendrites in the overlying molecular layer of the DG region where they receive synaptic connections mainly from the entorhinal cortex (EC) via the perforant pathway. A soma of a pyramidal cell rests in the cell layer of the CA region, while its dendrites extend across the strata from oriens layer (basal portion) to radiatum layer and molecular layer (apical portion) where they are making synapses with DG and EC.

In our previous modeling, we calculated the microscopic energy depositions and production of water radiolysis species in the vicinity of neuron structures using the Geant4/Geant4-DNA Monte Carlo radiation transport code [4-7]. In the present work, simplified models of a DG granule and a CA pyramidal cell were constructed from models of realistic neuron morphologies and adapted into standardized SWC file format. The total volumes of the simplified models were calculated with $11279.7 \mu \mathrm{m}^{3}$ for a DG granule cell and $31689.6 \mu \mathrm{m}^{3}$ for CA3/CA1 pyramidal cell. The total volumes of the realistic models of granule and pyramidal cell were found as $12455.5 \pm 4395.2 \mu \mathrm{m}^{3}$ and 34112.5 $\pm 9693.7 \mu \mathrm{m}^{3}$, which are estimated from all the hippocampal neurons in the Claiborne archive at the NeuroMorpho.Org repository. The realistic and simplified models of granule cell are represented as $451 \pm 228$ and 9 compartments of soma and dendrites. Pyramidal cell soma and dendrites are represented as $2259 \pm 563$ compartments for realistic model and 64 compartments for simplified model.

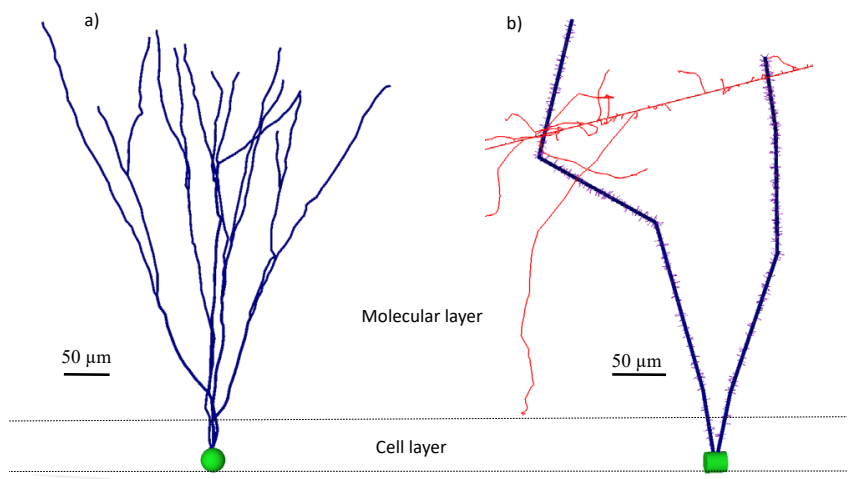

Figure 1. Realistic (a) and simplified (b) model of a granule cell in different layers of DG region of rat hippocampus. An example of a simulated track structure (red online, thin long lines in print) of 290 $\mathrm{MeV} / \mathrm{u}$ carbon ion crossing simplified model of a granule cell.

In order to build dendritic spines along the dendritic branches of the developed neuron models, we used the measured spine density $[8,9]$, which is defined as the total number of spines per unit length $(10 \mu \mathrm{m})$ of the total dendritic length. The spine density was found to be $8.2 \pm 0.8$ for the molecular layer of a DG granule cell, $8.0 \pm 0.6$ for the oriens layer and $11.3 \pm 0.5$ for the radiatum and molecular layers of the $\mathrm{CA} 3 / \mathrm{CA} 1$ pyramidal cell. Each spine is described by means of a small cylindrical volume that is randomly pointing perpendicular to the dendrite surface between $0-2 \pi$, and located on dendrites as a Gaussian distribution with given spine density. The spine sizes were generated from a Poisson distribution in a range between $0.3-0.76 \mu \mathrm{m}$ for diameter and $0.9-4.2 \mu \mathrm{m}$ for the total length, which were derived from literature $[10,11]$. In the simplified models, 810 spines for a DG granule cell and 3582 spines for a CA3/CA1 pyramidal cell were constructed using Poisson and Gaussian distributions with the above-mentioned spine densities. 


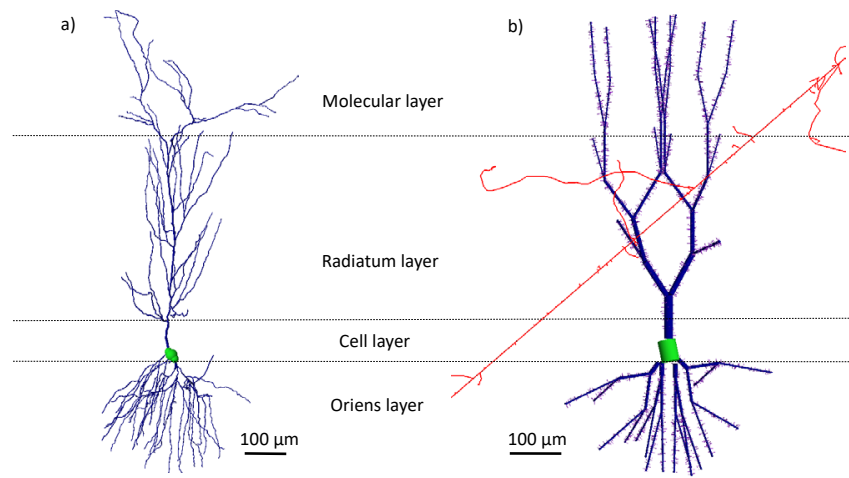

Figure 2. Realistic (a) and simplified (b) model of a pyramidal cell in different layers of CA3/CA1 region of rat hippocampus. An example of a simulated track structure (red online, thin long lines in print) of $290 \mathrm{MeV} / \mathrm{u}$ carbon ion crossing simplified model of a pyramidal cell.

\section{Comparison of simple and realistic neuron models for calculation of energy deposition and production of oxidative radical species}

The results of the three-dimensional construction of the developed neuron models with irradiation of particle track structure are illustrated in Figs 1 and 2. Similar distributions of the energy deposition events were obtained in the dendrites and soma of the two neuron models under an exposure to 290 $\mathrm{MeV} / \mathrm{u}$ carbon ion with a dose of $0.1 \mathrm{~Gy}$ (Figure 3). It should be noted that the error bars were estimated from the energy depositions in the individual neuron models in the Claiborne archive at the NeuroMorpho.Org repository.
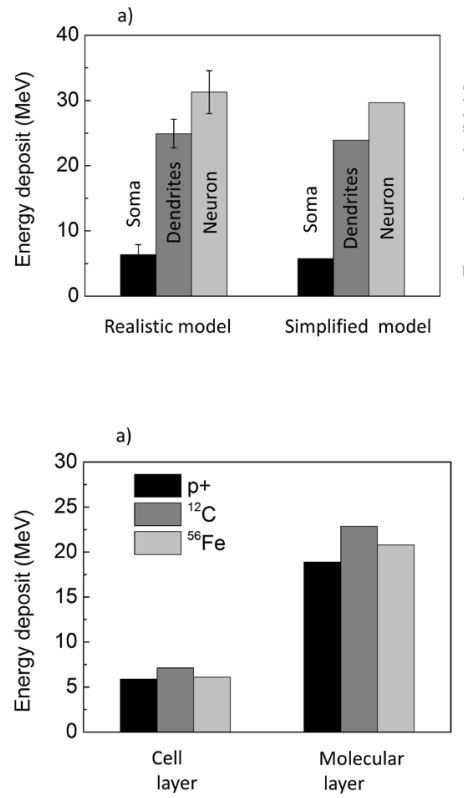

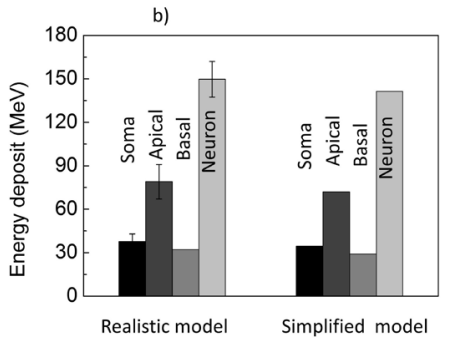

b)

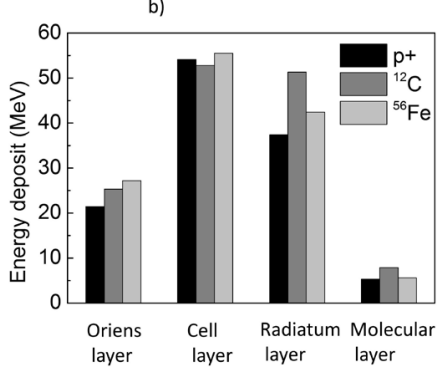

Figure 3. Total energy deposition in realistic and simplified model of a DG granule cell (a) and a CA3/CA1 pyramidal cell (b) under exposure to $290 \mathrm{MeV} / \mathrm{u}$ carbon ions with a dose of $0.1 \mathrm{~Gy}$.

Figure 4. Distribution of energy deposition in different layers of the simplified model of a DG granule cell (a) and CA3/CA1 pyramidal cell (b) under exposure to $170 \mathrm{MeV}$ protons, $290 \mathrm{MeV} / \mathrm{u}$ carbon and $600 \mathrm{MeV} / \mathrm{u}$ iron ions with the same dose of 0.1 Gy.

Under exposure to protons, carbon and iron ions with the same dose, the microscopic energy depositions in different layers of the granule and pyramidal cells were estimated and compared (Figure 4). We also calculated the molar concentration of molecules, which is the total number of oxidative radical species in a given volume of developed neuron models after exposure to different particles with the same dose (Figure 5). 

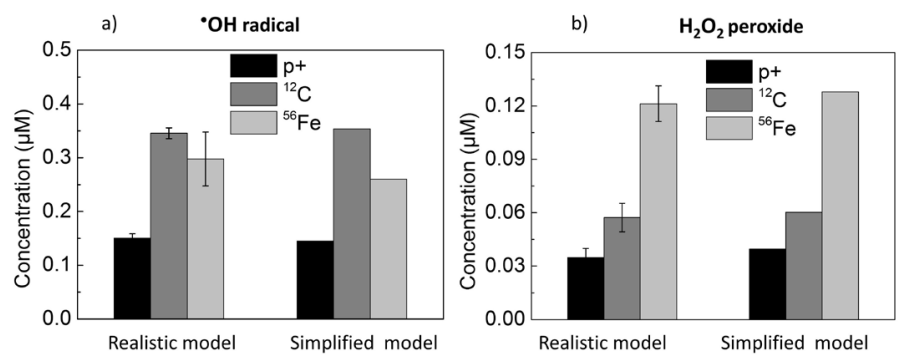

Figure 5. Total concentration of ${ }^{\bullet} \mathrm{OH}$ and $\mathrm{H}_{2} \mathrm{O}_{2}$ in models of a DG granule cell (a) and CA3/CA1 pyramidal cell (b) at 1 nanosecond after exposure to $170 \mathrm{MeV}$ protons, $290 \mathrm{MeV} / \mathrm{u}$ carbon and $600 \mathrm{MeV} / \mathrm{u}$ iron ions with the same dose of $0.1 \mathrm{~Gy}$.

\section{Conclusions}

This study presents the results of developments of simplified neuron models with properties equivalent to realistic neuron morphology for microdosimetric calculations, in order to investigate radiationinduced effects in the electrophysiology of hippocampal neurons. The geometric structure of a simple compartmental model and a complex model with realistic morphology extracted from experimental data were constructed in the Geant4/Geant4-DNA Monte Carlo transport code. This simulation was applied to build the uniform random distribution of spines along the dendritic branches of the developed neuron models, which were adapted into standardized SWC file format. Then, we compared the distribution of microscopic energy depositions and production of oxidative radical species in the simple and realistic neuron models under exposure to protons, carbon and iron ions with the same dose. The microdosimetric distributions were obtained to be similar in both the simplified and the realistic neuron models subject to irradiation. We should emphasize that the implementation of the realistic morphology meets difficulty to develop biological neural networks and the calculation of electrical activity requires much more computation time-consuming than in the case of the developed simplified models. New prospects are thus open for further development and understanding of the electrophysiological behaviour of irradiated hippocampal neurons on the basis of simplified models.

\section{Acknowledgements}

The study was conducted as part of the joint research (Protocol No. 4695-9-17/17) between the Laboratory of Radiation Biology of JINR and the National University of Mongolia. Authors acknowledge the financial support from the JINR Theme 04-9-1077-2015/2017.

\section{References}

[1] D. Greene-Schloesser et al., Front Oncol. 2, 73 (2012)

[2] A. G. Belyaeva et al., Neurochemical Journal 11, 2, 168-175 (2017)

[3] I.V. Sokolova et al., Radiat. Res. 183, 208-218 (2015)

[4] M. Batmunkh et al., J. Radiat. Res. Appl. Sci. 8, 498-507 (2015)

[5] M. Batmunkh, O.V. Belov, L. Bayarchimeg, and O. Lkhagva, Mong. J. Phys. 2, 317-323 (2016)

[6] O. V. Belov, M. Batmunkh, S. Incerti, and O. Lkhagva, Physica Medica 32, 1510-1520 (2016)

[7] L. Bayarchimeg, M. Batmunkh, O. Belov, and O. Lkhagva, EPJ Web of Conferences (this issue)

[8] E. Gould et al., J. Neurosci. 10 (4), 1286 (1990)

[9] E. Gould, M.D. Allan, and B.S. McEwen, Brain Res. 525 (2), 327-329 (1990)

[10] M. Papa, et al., J. Neurosci. 15, 1-11 (1995)

[11] A. Belly et al., J. Cell Sci. 123, 2943-2954 (2010) 